\title{
Féeries
}

Études sur le conte merveilleuX, XVII $-\mathrm{XIX}{ }^{\mathrm{e}}$ siècle

\section{Écritures européennes du conte d'Aladin et de la lampe merveilleuse}

European Writings of Aladdin and the Wonderful Lamp

\section{Margaret Sironval}

\section{(2) OpenEdition}

1 Journals

\section{Édition électronique}

URL : http://journals.openedition.org/feeries/121

DOI : $10.4000 /$ feeries. 121

ISSN : 1957-7753

\section{Éditeur}

UGA Éditions/Université Grenoble Alpes

\section{Édition imprimée}

Date de publication : 1 février 2005

ISBN : 2-84310062-3

ISSN : 1766-2842

\section{Référence électronique}

Margaret Sironval, «Écritures européennes du conte d'Aladin et de la lampe merveilleuse », Féeries [En ligne], 2 | 2005, mis en ligne le 19 décembre 2017, consulté le 07 septembre 2020. URL : http:// journals.openedition.org/feeries/121; DOI : https://doi.org/10.4000/feeries.121

Ce document a été généré automatiquement le 7 septembre 2020.

\section{(C) Féeries}




\title{
Écritures européennes du conte d'Aladin et de la lampe merveilleuse
}

\author{
European Writings of Aladdin and the Wonderful Lamp
}

Margaret Sironval

L'imaginaire se loge entre les livres et la lampe... On le puise à l'exactitude du savoir ; sa richesse est en attente dans le document. Pour rêver, il ne

faut pas fermer les yeux, il faut lire [...].

L'imaginaire ne se constitue pas contre le réel pour le nier ou le compenser ; il s'étend entre les signes, de livre à livre, dans l'interstice des redites et des commentaires ; il naît et se forme dans l'entre-deux des textes. C'est un phénomène de bibliothèque ${ }^{1}$.

DE TOUS LES CONTES DES « MILLE ET UNE NUITS », l'Histoire d'Aladin et de la lampe merveilleuse est celui dont l'histoire est une des plus singulières : par ses sources incertaines, (nul n'a encore trouvé de manuscrit arabe qui contienne le conte) ${ }^{2}$, et par les nombreuses réécritures et adaptations qu'il suscite encore aujourd'hui. En effet, l'Histoire d'Aladin est au palmarès des contes arabes pour l'importance de sa diffusion, en conte séparé du recueil des Mille et Une Nuits. La plupart de ces éditions sont destinées notamment à la littérature pour enfants et cela, dès le dix-huitième siècle, l'édition anglaise jouant un rôle de premier plan dans cette diffusion. L'Histoire d'Aladin est aussi le premier conte des Nuits diffusé par la littérature de colportage, le premier porté à la scène d'un théâtre, un théâtre londonien en l'espèce. Il est imbattable pour le nombre de versions orales recensées dans le monde : l'Enzyklopädie des Märchens ${ }^{3}$ compte 348 versions du conte réparties dans différentes aires culturelles. Enfin, il n'y a pas d'autres contes des Nuits, excepté l'Histoire de Shéhérazade, qui ait suscité autant d'écritures littéraires et d'adaptations théâtrales, musicales et filmiques. Résumons brièvement cette longue histoire : 


\begin{abstract}
Résumé
Pour obtenir une lampe qui lui donnera tous les pouvoirs, un magicien doit trouver un jeune garçon qui se chargera de descendre dans une certaine caverne, pour la prendre et la lui donner. Il compte bien se débarrasser de cet intermédiaire, une fois qu'il aura la lampe. Il cherche dans les rues d'une capitale de la Chine, un enfant malin, un peu voyou et c'est Aladin qu'il choisit en se déclarant son oncle, pour le mettre en confiance. Aladin descend dans la caverne remplie de trésors : il trouve la lampe, mais refuse de la donner au magicien avant d'être sorti. Le magicien l'enferme, croyant que la caverne serait son tombeau. Aladin y reste trois jours dans le noir, sans boire et sans manger. Mais, grâce à un anneau auquel est attaché un génie et que le magicien lui avait donné pour le protéger, Aladin revient chez lui emportant la lampe. Voulant aller la vendre pour se nourrir lui et sa mère, celle-ci la nettoie, et en la frottant, fait surgir un génie qui a le pouvoir d'exaucer tous les vœux. Grâce à la lampe, Aladin pourra prétendre épouser la princesse dont il est tombé amoureux. Mais la lampe est convoitée par le magicien et son frère qui chercheront tour à tour à la lui reprendre. Tous deux seront exécutés. Aladin finit par triompher de ces épreuves et gagne amour et fortune.
\end{abstract}

2 L'Histoire d'Aladin est fondée sur un thème capital : l'ascension sociale. Et ce roman d'ascension sociale parle à l'imaginaire de tous les lecteurs. En effet, rien ne désignait Aladin, qui ne se signale par aucun mérite, pour bénéficier d'un tel destin qui le mène de la rue au palais. Dès les premières lignes du récit, le conte arabe fait entrer le hasard dans l'existence du héros. Or, le sort revient à dénier les valeurs stables de ce monde en ignorant les conventions humaines : paresse, inattention, insolence, gaspillage juvénile, rien de bon ne devrait arriver à Aladin. Ajoutons que les notions de justice humaine n'y retrouvent pas leur compte comme elles le feraient avec un héros naturellement exemplaire. Or, Aladin est choisi, démontrant avec force, selon la formule, que «les voies du Seigneur sont impénétrables ».

3 Par cette allégorie de l'élection, Aladin se rapproche du héros du mythe : le choix d'Aladin par le magicien donne au conte valeur de mythe. En outre, la morale y trouve son compte : certes l'aventure du héros arabe peut-être lue comme un hymne au profit matériel, à l'enrichissement. Mais si l'on se place du côté de l'édition arabe et de la traduction anglaise du conte ${ }^{4}$, cette perspective n'est pas dévalorisante, la réussite en cette terre manifeste aux yeux de tous le rachat par Dieu. Une correspondance s'établit en quelque sorte entre l'élection divine et la réussite sur terre.

4 Les éléments merveilleux participent à la dimension mythique du conte. Les objets magiques, l'anneau et la lampe merveilleuse sont chargés d'ambiguïté : en effet, les génies qui leur sont attachés sont au service de celui qui les possède. L'efficacité bénéfique ou maléfique de ces objets est tributaire du personnage qui les détient. Entre les mains des magiciens du conte, la lampe merveilleuse sert l'idée d'un pouvoir sans bornes; aux mains d'Aladin, elle apporte au héros la possibilité d'accomplir le destin dont il rêve. Cette double situation aux desseins opposés a fasciné plusieurs auteurs. Il reste qu'à l'image du merveilleux des contes, celui du conte arabe entraîne également vers le lieu où va s'élaborer un destin, un destin inconnu et en cela, l'Histoire d'Aladin et de la lampe merveilleuse est bien à l'image du mythe : la rencontre avec l'étranger.

5 Étant donné le nombre de réécritures du conte pour la pantomime, pour les opérasféeries, pour le théâtre, les romans de gare, la littérature pour enfants, nous avons opéré une sélection de textes donnant une représentation diversifiée d'une littérature d'écrivains inspirés par l'Histoire d'Aladin. 
6 Nous avons retenu deux écritures issues d'un pays nordique, le Danemark : celle du poète et écrivain danois, Adam Oehlenschläger qui publie, pour le théâtre, en 1805, Aladin et la lampe merveilleuse, et celle de Hans Christian Andersen dont le premier récit de son recueil de contes, intitulé Le briquet, est une évocation danoise d'Aladin. Nous traiterons également de l'Aladin qui inspire à Marcel Schwob, la nouvelle, intitulée Sufrah géomancien, extraite de son recueil Vies imaginaires. Enfin Aladin inspire l'un des derniers livres écrits par le philosophe allemand, Ernst Jünger, intitulé : Le Problème d'Aladin.

\section{Adam Oehlenschläger (1779-1850)}

7 Oehlenschläger fait partie des poètes influencés par l'École romantique allemande. Il était très lié avec Goethe qui l'a encouragé sur la voie de la poésie. Il avait été en relation avec Ludwig Tieck et Henrik Steffens, Schelling et son ami le géologue et physicien H.C. Orsteed, réputé pour avoir découvert l'électro-magnétisme. Il était également lié avec Ernst Chladni (1756-1827) 5 qui, lui, est considéré comme « le père de l'acoustique moderne ». Oehlenschläger était très proche de Orsteed ${ }^{6}$. Le savant et le poète estimaient que les figures de Chladni sur les sons, les vibrations étaient la métaphore de l'esprit poétique. Et l'on retrouve ces métaphores dans l'œuvre maîtresse d'Oehlenschläger, Aladin et la lampe merveilleuse. Rappelons que Les Mille et Une Nuits étaient connues au Danemark, depuis la traduction des Mille et Une Nuits de Galland, en danois, en 1745. En 1805, Oehlenschläger publie au deuxième tome de ses Écrits poétiques, le drame épique en prose la Saga de Vaulundur inspirée par les Eddas du paganisme, et Aladin ou la lampe merveilleuse (Aladdin eller den forunderlige Lampe), pièce en cinq actes écrite en vers non rimés, à la manière de Shakespeare. Cette pièce a été traduite du danois en anglais, par Henri Meyer, en 1925.

\section{La pièce}

8 L'Aladin de Oehlenschläger s'ouvre avec la sombre figure du magicien qui veut la lampe pour obtenir richesse et pouvoir. Il est vêtu d'une longue robe noire, avec une ceinture et sur sa poitrine des broderies faites de mystérieuses figures. En réalité, Oehlenschläger reprend les figures acoustiques de Chladni, figures qui avaient permis au savant de mesurer l'effet produit par les sons au moyen d'un violon dont les cordes faisaient vibrer du sable qui, en tombant, sous la vibration de l'instrument, décrivait des figures parfaitement symétriques. Immédiatement, cette visualisation de l'invisible acquiert un statut métaphorique ${ }^{7}$. C'est ainsi que dans la pièce, le magicien est assis et dessine des marques sur le sable, essayant de les assembler en vain avec des lignes et des points. C'est alors que Oehlenschläger stigmatise l'impuissance du magicien à profiter lui-même de ce qu'il a découvert, la lampe merveilleuse qui donne tous les pouvoirs. Seul « un enfant de la nature » pourra s'en saisir et ce sera Aladin qui sera choisi pour devenir le détenteur du plus grand trésor qui soit au monde et pour lui rapporter une lampe. Car celle-ci ne peut être possédée que par un être pur et insouciant. La pièce suit en grande partie le conte d'Aladin. De nombreux passages en vers, insistent sur le fait que ce bonheur octroyé par les dieux dans cet état premier d'innocence sur lequel viendront se greffer par la suite les mérites individuels (la possession de la lampe merveilleuse), est amplement mérité par l'élu, Aladin. 
9 L'association entre le sombre magicien, les figures acoustiques de Chladni et l'innocence d'Aladin qui ne connaît pas les possibilités de la lampe, mettent en relief les tentatives d'appropriation du monde par l'homme. En bref, «Science sans conscience n'est que ruine de l'âme ». De cette âme justement qui remplit le propos développé dans la pièce danoise. De cette âme qu'il faut cultiver, quand on a été choisi, par un mérite individuel. L'objet de la quête, du côté d'Aladin, n'est pas la possession de la lampe, mais au travers elle, c'est bien de reconnaître l'omniprésence divine. Et dans un surplus de significations, pour Oehlenschläger, la lampe d'Aladin, c'est l'inspiration du poète, devant l'incapacité du magicien (l'homme de science) à saisir le merveilleux en s'obstinant à le chercher au travers de formules mathématiques, afin de trouver le lien entre la nature, Dieu et l'homme. Orsteed, dans une lettre à Oehlenschläger (qu'il appelait d'ailleurs Aladin), lui écrit ${ }^{8}$ : «les chemins séparés de l'art et de la science se rencontreront un jour» et Orsteed utilise la métaphore des figures acoustiques de Chladni pour conclure : "nous verrons un jour la fin de toute dissonance ». Mais ce n'est pas le seul mérite d'Orsteed : conçu comme un divertissement dramatique, Aladin exerce aussi une influence importante sur le développement du romantisme, et d'abord sur le disciple de Oehlenschläger, Hans Christian Andersen.

\section{Andersen (1805-1875)}

10 Andersen, auteur de pièces de théâtre et de romans, est surtout connu pour son recueil de contes. Celui qui nous occupe est le premier des 156 contes qu'Andersen ait écrit, intitulé Le Briquet ${ }^{9}$ dont la rédaction est inspirée par l'Histoire d'Aladin et les versions orales du conte qui circulaient déjà au Danemark à la fin du XviII siècle ${ }^{10}$.

11 Ce n'est pas un hasard si ce tout premier conte d'Andersen s'inspire d'Aladin. Les Mille et Une Nuits, comme le raconte Andersen dans sa biographie, avaient illuminé les veillées de son enfance ${ }^{11}$. Et surtout, il connaissait l'Aladin de Oehlenschläger, dont il était l'élève et le fervent admirateur. Quelques critiques lui reprocheront d'ailleurs d'avoir livré une " pâle imitation » du conte arabe.

Résumé

Un soldat rentrant de la guerre pour revenir chez lui rencontre une sorcière qui lui offre d'aller chercher un trésor enfoui dans des salles creusées sous le tronc d'un arbre. Elle ne veut rien de l'or qu'il prendra, juste le vieux briquet que sa grandmère y avait oublié. Avant de lui donner ce briquet, le soldat lui demande ce qu'elle veut en faire. La sorcière refuse de le lui dire; il la décapite. Muni de tous ses trésors, il se rend à la ville dans laquelle il aperçoit la fille du roi dont il tombe amoureux. Avec l'or il mène grand train. Mais le trésor s'épuise. Il n'a plus un sou, pas même pour acheter une chandelle. Il se souvient du briquet qui était bien sale avec un bout de chandelle collé dessus. En voulant l'allumer, la flamme fait jaillir un chien énorme qui lui demande ce qu'il veut. Il demande la princesse. Le chien la transporte près du soldat plusieurs nuits de suite jusqu'au jour où le soldat est démasqué grâce à une ruse pour le retrouver (des grains de seigle). Il est mis en prison. Là, il s'arrange pour qu'on lui apporte son briquet et rétablit la situation à son avantage : au moment où il va être pendu, il allume sa dernière pipe, les trois chiens surgissent, se saisissent des juges, du roi de la reine et de la cour, les jettent en l'air et leur corps disparaissent. Le soldat épouse la princesse et devient roi.

Le succès d'Aladin au Danemark, évoqué plus haut, est provoqué par l'idée, sous-jacente au conte arabe évoqué plus haut, qu'un vaurien qui croit en Dieu peut être récompensé en étant choisi par Lui, développant ainsi la croyance en un bonheur miraculeux ${ }^{12}$. Mais 
ajoutons que dans ce cas, le héros ne parvient à s'élever dans la société que s'il y a mérite individuel. C'est ce qui avait aussi séduit Oehlenschläger dans Aladin.

L'inspiration tirée du conte arabe par Andersen correspond, elle, à l'idée que se faisait une classe bourgeoise montante qui avait suivi le mouvement romantique au travers de ce que la littérature danoise appelle «le motif d'Aladin» d'après l'idée que Oehlenschläger exprima dans sa pièce en vers Aladin ou la lampe merveilleuse :

Cela concerne la théorie selon laquelle certaines personnes sont choisies par la nature, par Dieu ou par des dieux, pour atteindre la grandeur et que rien ne peut parvenir à les arrêter, quels que soient la faiblesse ou le mal dont ils puissent souffrir. La possibilité d'être choisi pour devenir grand, en dépit des apparences, prit une signification particulière au Danemark, au début du XVIII siècle, où l'aristocratie dominait et où la population cherchait une échappatoire à un présent sordide. C'est ainsi que le concept d'Aladin prenait de nouvelles proportions : il n'était pas seulement utilisé comme thème littéraire mais pouvait s'appliquer aux individus $^{13}$.

14 Le rapport qui peut être établi entre le personnage du Briquet d'Andersen et Aladin est essentiellement fondé sur l'idée d'une élection qui échappe aux lois humaines. En effet, comme Aladin, le soldat est choisi par la vieille sorcière sans que l'on donne de raison évidente. Il en diffère cependant par une qualité tout à fait remarquable : ce n'est plus un tout jeune garçon innocent et insouciant, mais un soldat qui, par son statut, n'est pas assimilé à un vaurien. Cette transformation du héros en militaire pourrait trouver une explication par le contexte historique de l'époque pendant laquelle se sont répandues les versions d'Aladin dans les pays du Nord. Les guerres napoléoniennes avaient mis au premier rang la figure du héros soldat qui n'avait pas toujours la chance d'obtenir en récompense de ses exploits un royaume et le titre de roi. En outre, la conscription, qui dure jusqu'en 1870, coïncide avec un type d'expérience ou le hasard et la chance ont leur part: "On nous fait tirer au sort pour nous conduire à la mort ", disaient les soldats. Comme eux, Aladin est choisi par le magicien pour accomplir une certaine tâche et mourir. Mais les effets du hasard s'avèrent parfois bénéfiques.

ché d'Andersen, le choix du héros soldat incarnait les possibilités de promotion illimitées dont pouvait raisonnablement rêver un homme de talent ambitieux. L'auteur, lui-même, était un fervent admirateur du modèle napoléonien ${ }^{14}$. Le changement de statut du héros dans le conte nordique conserve avec l'idée de l'élection, le thème de la promotion sociale.

Dans le Briquet, Andersen introduit le soulèvement populaire que l'on rencontre dans le conte d'Aladin ${ }^{15}$ en modifiant notablement l'issue de ce conflit. L'assentiment de la communauté signalée dans l'un et l'autre conte ne se manifeste pas dans les mêmes conditions

17 Aladin par ses libéralités s'est acquis une grande popularité. Il a fait ses preuves, il a épousé la princesse, ses qualités le désignent comme le meilleur prétendant au trône pour succéder naturellement au sultan. Il faut noter que la réussite sociale d'Aladin ne devient effective qu'en dehors des aides surnaturelles, après avoir reçu le témoignage de confiance du sultan et l'accord de la communauté.

18 Le petit soldat d'Andersen n'a, lui, d'autre soutien que l'instrument magique du pouvoir qu'il a acquis et dont il dispose sûrement puisqu'il a tué la sorcière. Il n'a d'autre solution que de mettre hors jeu le roi, la reine et sa cour, qui s'opposent à son mariage avec la princesse, afin de prendre leur place. Le petit soldat conquiert le pouvoir par un coup d'État, avec l'aide de ses auxiliaires magiques (les trois chiens), assujettissant le 
roi et sa cour et convaincant le peuple que cet acte de force (exécuter le roi et la reine) le désigne comme le plus apte.

Les deux réécritures suivantes opèrent un renversement fondamental de l'utilisation de la lampe et de sa fonction par rapport à la composition danoise d'Oehlenschläger tournée vers l'allégorie de l'élection et du pouvoir poétique. Le conte d'Andersen fait la transition avec ces textes, l'objet merveilleux (le briquet) devenant l'instrument d'un pouvoir à conquérir afin que le soldat réalise son ascension sociale. Les deux écrivains, Marcel Schwob et Ernst Jünger, détournent les vertus du pouvoir délivré par les objets magiques: ainsi, la possession de la lampe ou de l'anneau n'est plus d'exercer leur action sur la vie de celui qui les possède, mais d'obtenir grâce à eux, le pouvoir sur la mort.

\section{Marcel Schwob}

Dans les Vies imaginaires, Marcel Schwob écrit un petit conte intitulé: Sufrah géomancien ${ }^{16}$. L'écrivain ne croit pas à la mort des magiciens, comme il ne peut envisager la disparition du mal sur terre. Des deux objets merveilleux, Schwob s'intéresse au pouvoir de l'anneau ${ }^{17}$ et réécrit l'histoire dans cette perspective :

21 L'histoire d'Aladdin, conte par erreur que le magicien africain fut empoisonné dans son palais et qu'on jeta son corps noirci et craquelé par la force de la drogue aux chiens et aux chats; il est vrai que son frère fut déçu par cette apparence et se fit poignarder, ayant revêtu la robe de la sainte Fatima ; mais il est certain néanmoins que Moghrabi Sufrah (car c'était le nom du magicien) s'endormit seulement par la toute-puissance du narcotique, et s'échappa de l'une des vingt-quatre fenêtres du grand salon, pendant qu'Aladdin embrassait tendrement la princesse. Ayant ainsi échappé à la mort, Sufrah se retrouve assis dans le désert sans rien à manger ni à boire. Au moyen de la géomancie, il demande s'il est destiné à périr ou pas dans le désert. Assuré de ne pas mourir sur-le-champ, Sufrah se mit à réfléchir. Il n'avait pas l'espoir de reconquérir la lampe, qui avait été transportée avec le palais dans le centre de la Chine. Cependant il songea que jamais il n'avait recherché quel était le véritable maître du talisman et l'ancien possesseur du grand trésor et du jardin aux fruits précieux.

C'est à cet endroit que la question de la lampe ressurgit: comme ses prédécesseurs, Marcel Schwob s'interroge sur l'origine puis sur le devenir de la lampe merveilleuse. Rappelons que le conte aborde le sujet à la fin du récit : Aladin demande au génie de la lampe de lui apporter l'œuf de Rokh qui pend dans la salle d'apparat de son palais. Cette demande équivaut à demander la mort du roi des génies. Or le roi des génies dans les légendes et dans la littérature arabes, est Salomon. Et Schwob, peut-être parce qu'il connaissait le rôle joué par le grand roi dans la littérature arabe, fait en tout cas dériver son récit vers l'histoire de Salomon. Et écrit-il: «Sufrah apprend alors que la lampe merveilleuse avait fait partie du trésor du roi Salomon ». C'est alors qu'une nouvelle figure de géomancie lui révèle que son tombeau se trouve non loin de l'endroit où il est lui-même, et que «le roi porte encore à son doigt son sceau tout-puissant qui donne l'immortalité terrestre: si bien qu'il devait être endormi depuis des myriades d'années ». Ainsi, Sufrah se met en route pour arriver dans un lieu montagneux où il découvre l'entrée du souterrain. Comme Aladin dans le conte, il soulève une pierre munie d'un anneau de bronze. Il s'engage alors dans un long couloir jusqu'à une porte de fer gardée par « deux chiens de feu ${ }^{18} »$. Sufrah finit par entrer et se trouve dans un 
labyrinthe composé de multiples salles. Il les parcourt jusqu'à parvenir dans une chambre carrée où sur un lit "fait d'un seul diamant taillé », est étendue " une forme vieille, à barbe blanche, le front ceint d'une couronne [...]. Sur la main pendante du roi Salomon, Sufrah vit briller le grand sceau », et alors "rampant jusqu'au lit, Sufrah fit glisser l'anneau et le saisit ». Immédiatement le corps du roi Salomon tombe en poussière, réduit à une petite poignée d'ossements blancs. Terrassé à son tour, le magicien prend la place du roi, le sceau à son doigt et « tombe dans l'assoupissement de l'immortalité terrestre». Pour finir, «La porte de fer retomba sur le couloir de porphyre et les chiens de feu commencèrent à veiller le géomancien immortel ».

Ainsi, jusque dans la mort, le magicien cherche à être un maître puissant. En volant l'anneau du roi Salomon, il conquiert le sommeil d'immortalité. Une fois encore, dans cette écriture littéraire du conte d'Aladin, Marcel Schwob cherche dans les résonances d'un texte ce qui pourrait le prolonger, lui faire suite, ou encore susciter une autre façon de raconter le récit. Cette fouille du passé reflète bien l'activité de Schwob, toujours prêt à chercher dans un texte ce qu'il a d'irréductible, de secret et auquel il pourrait donner vie en se saisissant des métaphores qu'ils suggèrent, par un autre texte $^{19}$.

\section{Ernst Jünger}

\section{.} de Pologne). À un moment de sa vie, il se trouve totalement démuni. Après diverses tentatives pour s'en sortir, il se résout à aller voir son oncle Fridolin pour lui demander un travail dans son entreprise funéraire. Friedrich est chargé de contacter les familles des défunts pour organiser les funérailles. C'est alors qu'il rencontre des amis qui partent du constat que la mort est caduque puisque les tombes n'ont pas l'assurance de la perpétuité. Ils décident ensemble de monter une société qui gérera la mort à l'échelle planétaire. Le projet grandiose est de faire un cimetière où les gens reposent éternellement. Ils fondent une nouvelle société « Térestra » et cherchent un terrain pour l'installer; c'est en Cappadoce que la société s'établit. Friedriech est nommé directeur de l'entreprise. La réussite de la société est telle que Friedrich est en butte à des hallucinations au cours desquelles il rencontre Farès (alias le magicien). Dans son délire, il s'aperçoit que la caverne d'Aladin jouxte les cavernes de Cappadoce et que la lampe qui est dans la caverne d'Aladin est en étain tandis que la lampe qui éclaire les locaux de l'entreprise est en uranium. L'entreprise continue à se développer à tel point qu'il faut engager un nouveau dirigeant. Friedrich reçoit de Chine une candidature qui correspond étrangement à ce qu'il recherche. Elle est signée Farès, lequel lui donne rendez-vous. Friedrich (Aladin) va à sa rencontre tout joyeux, à l'idée de lui confier la direction de l'entreprise et de se libérer ainsi de son fardeau (la lampe).

Pour reprendre l'image de Jünger à propos de la lampe, "le chèque en blanc » que représentent sa possession et son utilisation, comporte les plus grands risques. Tous les vœux sont possibles. Cette licence induit la démesure et celui qui possède la lampe sera exposé à la tentation d'exercer le pouvoir qu'elle donne, à l'égal d'un démiurge ${ }^{21}$. Dans Le Problème d'Aladin, la possession d'un tel cadeau semble n'offrir qu'une alternative : ou le héros l'exploite «à la folie» et l'on aboutit aux pires extrémités de la prise d'un 
pouvoir sans partage ; ou, dans le meilleur des cas, cette «folie » n'est imaginable que dans l'art parce qu'elle sert de révélateur à un don artistique ${ }^{22}$. Autre traitement remarquable du mythe d'Aladin: la possession de la lampe merveilleuse n'est concevable que dans l'optique de l'artiste, c'est-à-dire de la création. Rappelons ici, que cette valeur connotative de la lampe avait déjà pris forme, pour la première fois, dans l'Aladin d'Oehlenschläger pour qui la lampe s'apparente à l'inspiration du poète et a pour rôle de signifier la poésie.

Du côté du magicien, la démesure que lui offre la possession de l'objet merveilleux ne connait pas de frein. Il s'agit bien pour le magicien de la tentation de l'hybris, du désir d'obtenir plus que son droit, et « toujours [...] la démesure ancienne [...] fait naître une démesure neuve ${ }^{23}$ ». Face à cette situation, Aladin oppose une attitude raisonnable, trop peut-être. C'est en tout cas ce que Ernst Jünger manifeste : "Quelque chose vole à moi, la richesse afflue. Je dois décider de la façon dont je la maîtriserai. Mais cela ne doit pas être à la manière d'Aladin » ou encore, plus précisément, « Aladin pouvait se borner au confort; il n'a pas non plus avec Boudour connu autre chose qu'un heureux mariage. Ainsi procèdent les esprits simples; ils demeurent à l'abri d'une tentation plus forte ${ }^{24}$ ». Pour le magicien, la lampe ne signifie rien d'autre que l'accession à un pouvoir sans bornes. Avec le court extrait qui suit, Jünger est tout près du mythe et de la dimension prométhéenne du conte :

La lampe d'Aladin était faite d'étain ou de cuivre, peut-être d'argile seulement. Le texte de Galland ne nous dit rien là-dessus - nous apprenons seulement qu'elle était suspendue à la voûte d'une grotte. On ne l'allumait pas, mais on la frottait pour que le génie apparaisse. Il pouvait en une nuit édifier des palais ou ravager des villes, selon que l'ordonnait le maître de la lampe. Elle garantissait la souveraineté jusqu'aux limites du monde connu de la Chine jusqu'à la Mauritanie, Aladin préférait l'existence d'un petit despote. Notre lampe est faite d'uranium. Elle pose le même problème : l'afflux de puissance titanique ${ }^{25}$.

Marcel Proust associait les images des Mille et Une Nuits avec l'univers d'origine, pour lui les Nuits faisaient partie du mythe et des illuminations qui mènent à la création. La leçon proustienne des Mille et Une Nuits est celle de la métaphore qui fait voir dans une chose, une autre chose. Or, ces différentes écritures européennes du conte d'Aladin, nous offrent quelques exemples de la variété des métaphores que le récit a inspirées. Rappelons encore Balzac, qui s'était engagé dans une lecture symbolique des contes, et qui, en son temps, avait vu dans la magie des Nuits une métaphore de l'art. Nous ne saurions mieux conclure qu'en le citant: "Combien de Lampes merveilleuses faut-il avoir maniées avant de reconnaître que la vraie Lampe merveilleuse est ou le hasard, ou le travail, ou le génie ${ }^{26}$ ?»

\section{NOTES}

1. Extrait de M. Foucault, «La Bibliothèque fantastique », dans Travail de Flaubert (1967), Paris, Seuil, « coll. Points », 1983.

2. L'Histoire d'Aladin et la lampe merveilleuse appartient au noyau le plus ancien des contes des Mille et Une Nuits. Aladin fait partie des récits racontés oralement à Galland, par son correspondant 
syrien, le moine maronite Hanna. Pour Aladin, comme pour l'histoire d'Ali Baba, il n'y a pas de texte arabe connu. Aladin a été publié par l'imprimeur-libraire Florentin Delaulne, à Paris en 1712. C'est un des contes le plus long du recueil dans l'édition Garnier-Flammarion, Paris, 1965, t. III, (169 pages). Dans l'édition princeps, il figure aux tomes $9^{\mathrm{e}}$ et $10^{\mathrm{e}}$, sur les 12 tomes de la traduction des Mille et Une Nuits par A. Galland, qu'il a faite pour la plus grande partie d'après le manuscrit arabe des Mille et Une Nuits, le plus ancien connu à ce jour, déposé à la Bibliothèque nationale et daté $\mathrm{du} \mathrm{XIV} / \mathrm{Xv}^{\mathrm{e}}$ siècle. Pour un commentaire détaillé sur les sources d'Aladin ou la lampe merveilleuse, voir J. Sadan, «Background, date and meaning of the Story of the Alexandrian Lover and The Magic lamp, A little-known story from Ottoman times with a partial resemblance to the story of Aladdin ", dans Quaderni di Studi Arabi, n 19, Université de Foscari, Venise, 2001.

3. K. Kanke, «Aladdin» dans Enzyklopädie des Märchens, vol. I, Berlin et New York, 1977, p. 239-247.

4. Les adaptations des éditions anglaises du conte pour la littérature pour enfants valorisent cet aspect de l'histoire.

5. M.D. Waller, Chladni figures : A study in Symmetry, London, G. Bell and Sons, Londres, 1961.

6. J.-L. Greenway, "Orsted's Theory of light and Oehlenschlager's Aladdin », XVII, Studienkonferenz der International Association for Scandivian Studies, ed. Oskar Bandle, Zürich : Basel, Helbing et Lichtenhahn, 1988, p. 376-381.

7. Napoléon citait les travaux de Chladni en ces termes : "Chladni rend les sons visibles ", dans M. Waller, ouvr. cité, p. 2.

8. H.C. Oersted, Correspondance avec divers savants, (3 vol.), Ed. M.C. Harding, Copenhague, 1920.

9. H.C. Andersen écrit ses 156 contes entre les années 1835 et 1875. Le Briquet est publié en 1835. H.C. Andersen, "Le Briquet", dans Contes danois traduits par A. Soldi, avec une notice biographique de Xavier Marmier, illustré de quarante vignettes de Bertall, Hachette, Paris, 1856, p. 27-39. Voir aussi, Contes danois de H.C. Andersen, traduit, présenté et annoté par Régis Boyer, Paris, Gallimard, 1992.

10. La seconde traduction des Mille et Une Nuits en danois a été faite par Rasmussen (Jens Lassens), en 1824, d'après la première édition du texte arabe de Calcutta (1814). À propos de l'édition de Calcutta, voir Elisseeff (N), Thèmes et motifs des Mille et Une Nuits, ouvr. cité, p. 65-66. On trouve également une version analogue de l'Histoire d'Aladin et du Briquet dans Contes d'enfants et du foyer (1812) des frères Grimm, sous le titre, La Lumière bleue. Voir aussi, «La lumière bleue " dans Jacob et Wilhelm Grimm, Les contes, texte français et présentation par Armel Guerne, Paris, Flammarion, 1967, (réed. 1986), p. 670-675.

11. «Mon père ne se sentit pas chez lui dans son milieu, il n'avait pas grand goût pour le travail, aussi, le soir, lisait-il à haute voix Holberg et Les Mille et Une Nuits ", dans Hans Christian Andersen, Cuvres, textes traduits, présentés et annotés par Régis Boyer, Paris, Gallimard, 1992, Biographie, $\mathrm{I}^{\mathrm{re}}$ section, chap. III, p. 9.

12. Michelet, en son temps, voyant se multiplier les romans vendeurs d'illusions, écrit dans Nos fils: «Depuis le 2 décembre (1851), le grand flot des romans qui nous ont envahis, est dominé surtout par l'idée d'aventure, de bonheur improbable, de loterie grossière, l'idée californienne de gros lot et de lingot d'or. Toujours la foi aveugle au miracle, au hasard, au coup d'état du sort, qui dispense d'effort, de travail, de persévérance ». Citation extraite de Martyn Lyons, Le Triomphe du Livre, une histoire sociologique de la lecture dans la France du XIX siècle, Paris, Promodis, 1987 p. 191.

13. Ce commentaire est repris de N. Glyn Jones, Denmark, New York, Praeger, 1970, (p. 17-129), et repris dans J. Zipes, Les Contes de fées et l'art de la subversion, étude de la civilisation des mours à travers un genre classique, la littérature pour la jeunesse, traduit de l'anglais par François-Ruy-Vidal, Paris, Payot, 1986, p. 101.

14. À ce sujet, Andersen relatant son voyage en France, écrivait : «Avec un religieux respect, je franchis le seuil de la chambre de Napoléon. Je touchai de la main l'estrade où son pied s'était posé ; j'effleurai de mes doigts l'oreiller où avait dormi le héros de mon père et de mon enfance. 
Si j'avais été seul, je serais tombé à genoux ", dans, Hans Christian Andersen, Le Conte de ma vie, traduit du danois par Cécile Lund et Jules Bernard, préface de Jean Cassou, Paris, Stock, 1930, p. 106.

15. Le thème sera développé plus tard par Hasek Jaroslav (1883-1923), qui écrit entres les années 1917 et 1923, le fameux roman, Le Brave Soldat Chvëik dont Andersen, nous semble-t-il, avait donné l'ébauche du personnage dans son conte « Le Briquet ». Le Brave Soldat Chveik, traduit du tchèque par Henry Horejsi. Préface de Jean-Richard Bloch. Paris, Club français du Livre, 1964. Le roman a été repris pour le théâtre par Bertold Brecht qui écrit la pièce aux États-unis entre 1943 et 1954. Schweyk de Brecht sera représenté plus tard, en 1957.

16. Marcel Schwob, «Sufrah géomancien » dans Vies imaginaires, Paris, Crès, 1921, p. 74-79.

17. On a tendance à oublier qu'Aladin a reçu deux objets merveilleux : avant la lampe, il a reçu l'anneau, donné par le magicien à Aladin avant de descendre dans le souterrain, pour le protéger des dangers qui l'attendent. Dans le conte, l'introduction de cet anneau et du génie qui lui est attaché remplit une fonction bien précise : par trois fois, il évite une mort certaine à Aladin et lui permet d'être transporté ou il le demande.

18. Ces chiens nous rappellent ceux qu'Andersen avait placé, en remplacement des génies, dans son conte Le Briquet.

19. Sur l'œuvre de Marcel Schwob, Agnès Lhermitte, Palimpseste et merveilleux dans l'œuvre de Marcel Schwob, Paris, H. Champion, 2002.

20. Ernst Jünger, Le Problème d'Aladin, trad. par Henri Thomas, éd. Ch. Bourgois, Paris, 1983. Jünger écrit ce roman à l'âge de 88 ans, juste avant Les Ciseaux qui seront publiés en 1990.

21. Rappelons une fois encore que la lampe ne fonctionne que parce que l'exécutant qui lui est attaché, le génie, en est l'esclave. Or, lorsque les réécritures changent la position du génie, le libérant de l'objet merveilleux, il n'y a plus à craindre ses pouvoirs exorbitants. Le génie libéré de ses chaînes pourra choisir son maître et ne plus obéir systématiquement à celui qui détient la lampe merveilleuse. Ce choix revient au problème posé par l'anneau dans Tolkien, qui rend dépendant de lui ceux qui le possèdent.

22. Voir Le Problème d'Aladin, ouvr. cité, Le mystérieux Pharès (alias le magicien du conte) agit auprès du héros comme le révélateur d'un don. Homme d'affaires talentueux et nihiliste, sa réussite dans une entreprise de pompes funèbres est telle qu'il est appelé à la diriger. Le héros est pris à son propre piège. Il doit croire en lui et faire la preuve de ce qu'il avance.

23. Voir Raymond Trousson, Le thème de Prométhée dans la littérature européenne, 2 tomes, Genève, Droz, 1964, à propos du Prométhée enchaîné d'Eschyle, p. 33.

24. Le Problème d'Aladin, ouvr. cité, p. 122.

25. Le Problème d'Aladin, ouvr. cité, p. 116

26. Honoré de Balzac, Études sur les mœurs. 1er Livre. Scènes de la vie privée (tome 4), Honorine.

\section{RÉSUMÉS}

Les Mille et Une Nuits, recueil de contes, sans auteur désigné, libre de copyright, sont apparues comme une manne pour les éditeurs et pour les écrivains : elles ont suscité maintes adaptations dans les livres, au théâtre, au cinéma, dans la mode, la publicité et les beaux-arts. Les réécritures littéraires de l'Histoire d'Aladin et la lampe merveilleuse témoignent de cette diversité d'inspiration depuis la publication du conte en France au XVIII ${ }^{\mathrm{e}}$ siècle. 
The Thousand and One Nights, a collection of tales with no known author and free of all copyright, were a blessing for writers and publishers alike. The tales gave birth to innumerable adaptations in books, plays and films, as well as in fashion, advertising and the fine arts. The many rewritings of Aladdin and the Wonderful Lamp, which followed the initial publication of the tale in France in the $18^{\text {th }}$ Century, testify to this tremendous diversity.

\section{AUTEUR}

MARGARET SIRONVAL

CNRS (Paris) - Institut de recherche et d'histoire des textes 